\title{
GLOBAL HYPOELLIPTICITY OF A MATHIEU OPERATOR
}

\author{
MASAFUMI YOSHINO
}

(Communicated by Barbara L. Keyfitz)

\begin{abstract}
We give a necessary and sufficient condition for the global hypoellipticity of a Mathieu operator on the torus $\mathbb{T}^{d}$ in terms of continued fractions. It is not hypoelliptic, nor does it satisfy a controllability condition, a Hörmander condition, or a Siegel condition. But it is still globally hypoelliptic (cf. [1, 3]).
\end{abstract}

\section{INTRODUCTION}

Let $d \geq 2$ be an integer, and let $\mathbb{T}^{d}=\mathbb{R}^{d} / 2 \pi \mathbb{Z}^{d}$ be a $d$-dimensional torus. We denote the variable in $\mathbb{T}^{d}$ by $x=\left(x_{1}, \ldots, x_{d}\right)$, and by $\mathscr{D}^{\prime}\left(\mathbb{T}^{d}\right)$ and $C^{\infty}\left(\mathbb{T}^{d}\right)$ the set of distributions and the smooth functions on $\mathbb{T}^{d}$, respectively. Let $P$ be a differential operator on $\mathbb{T}^{d}$. We say that $P$ is hypoelliptic in $\mathbb{T}^{d}$ if, for any domain $\Omega \subset \mathbb{T}^{d}, P u \in C^{\infty}(\Omega)$ and $u \in \mathscr{D}^{\prime}(\Omega)$ imply $u \in C^{\infty}(\Omega)$. On the other hand, $P$ is globally hypoelliptic in $\mathbb{T}^{d}$ if $u \in \mathscr{D}^{\prime}\left(\mathbb{T}^{d}\right)$ and $P u \in C^{\infty}\left(\mathbb{T}^{d}\right)$ imply $u \in C^{\infty}\left(\mathbb{T}^{d}\right)$.

There is much research to characterize the difference between hypoellipticity and global hypoellipticity. One trend is, as for second order equations, to characterize it by the property of certain vector fields determined by the equation (cf. [3]). But this way cannot explain all globally hypoelliptic operators because we have two quite different examples. One is Greenfield's example $Q=\left(\partial / \partial x_{1}-\tau \partial / \partial x_{2}\right)^{2}$ where $\tau$ is an irrational number. $Q$ is globally hypoelliptic in $\mathbb{T}^{2}$ if and only if $\tau$ satisfies a Siegel condition (cf. [1]). The other is a so-called Mathieu operator, $P \equiv-\left(\partial / \partial x_{1}\right)^{2}+2 \cos x_{1}$. Though $P$ does not satisfy a Siegel condition, it is globally hypoelliptic in $\mathbb{T}^{2}$ (cf. [4]). We note that the result in [4] is only a sufficient condition for the global hypoellipticity, and it is not applicable to the operators $P-\lambda, \lambda \in \mathbb{C}$.

In this paper, we shall give a necessary and sufficient condition for the global hypoellipticity of $P-\lambda$. As a result we shall show the close connection between global hypoellipticity and the positions of zeros and poles of a certain meromorphic function defined by a continued fraction. In fact, let us define a

Received by the editors February 14, 1989.

1980 Mathematics Subject Classification (1985 Revision). Primary 35HXX; Secondary 35JXX.

Key words and phrases. Hypoellipticity, global hypoellipticity, continued fractions. 
meromorphic function $T(z)$ by a continued fraction

$$
T(z)=K_{n=1}^{\infty}\left((-1) / b_{n}\right), \quad b_{n}=z-n^{2}
$$

(as for the notations and the existence of the right-hand side of (1), we refer to [2]), and let us set $P=-\left(\partial / \partial x_{1}\right)^{2}+2 \cos x_{1}$. Then we have

Theorem. The following statements are equivalent.

(a) $P-\lambda$ is globally hypoelliptic in $\mathbb{T}^{d}$.

(b) $P-\lambda: C^{\infty}(\mathbb{T}) \rightarrow C^{\infty}(\mathbb{T})$ is injective.

(c) $\lambda$ is not a pole of $T(z)$ and $2 T(\lambda) \neq-\lambda$.

If $\lambda=0$, then condition (c) says that $T(z)$ does not have a pole or a zero at the origin. This is satisfied in case $b_{n}=-n^{2}$ by the lemma which follows (cf. [2]). Hence we have (cf. [4])

Corollary. The Mathieu operator $-\left(\partial / \partial x_{1}\right)^{2}+2 \cos x_{1}$ is globally hypoelliptic in $\mathbb{T}^{d}$.

\section{Proof of THE THEOREM}

We prepare a lemma.

Lemma. Let the sequences $\left\{A_{n}\right\}$ and $\left\{B_{n}\right\}$ be the solutions of the three-term recurrence relations

$$
y_{n}=b_{n} y_{n-1}-y_{n-2}, \quad b_{n}=\lambda-n^{2}
$$

for $n \geq-1$ with the initial conditions $A_{-1}=1, A_{0}=0, B_{-1}=0, B_{0}=1$, respectively. Then we have

(a) The determinant formula, $A_{n} B_{n-1}-A_{n-1} B_{n}=-1$, holds for $n=0,1$, $2, \ldots$

(b) $\left\{A_{n}\right\}$ (resp. $\left\{B_{n}\right\}$ ) is exponentially decreasing if and only if $T(\lambda)=0$ (resp. $T(\lambda)=\infty)$. If $\left\{A_{n}\right\}$ (resp. $\left.\left\{B_{n}\right\}\right)$ is not exponentially decreasing, then it is exponentially growing.

(c) Suppose $T(\lambda) \neq \infty$. Then there exist $K>1$ and $n_{0}$ such that, for $n \geq n_{0}$,

$$
\left|A_{n} B_{n}^{-1}-T(\lambda)\right| \leq(K-1)^{-1}\left|B_{n}\right|^{-2} .
$$

The lemma follows from [2, Theorem B.4].

Proof of theorem. Since (a) trivially implies (b), we shall show that (c) follows from (b). Let us substitute the Fourier expansion of $u, u=\sum_{\eta} u_{\eta} e^{i \eta x}$, into the equation $(P-\lambda) u=0$. Then we have recurrence relation (2) for $y_{n} \equiv u_{\left(n+1, \eta^{\prime}\right)}$, $b_{n}=\lambda-n^{2}$ for $n=0, \pm 1, \pm 2, \ldots$ If $T(\lambda)=\infty$, then it follows from part (b) of the lemma that the sequence $y_{n}=B_{n}(n \geq-1),=B_{-n-2} \quad(n \leq-1)$ gives a solution of (2) which is exponentially decreasing as $n \rightarrow \pm \infty$, which contradicts (b). On the other hand, if $|T(\lambda)|<\infty$ and $2 T(\lambda)=-\lambda$, then 
we can easily see, from part (c) of the lemma, that $y_{n}=\alpha\left(\eta^{\prime}\right)\left(A_{n}-T B_{n}\right)$ $(n \geq-1) ; y_{n}=\alpha\left(\eta^{\prime}\right)\left(A_{-n-2}-T B_{-n-2}\right) \quad(n<-1)$ gives a solution of $(2)$ which is exponentially decreasing as $n \rightarrow \pm \infty$, a contradiction to (b).

Next we shall show that (c) implies (a). By substituting the expansions of $u$ and $f, f=\sum_{\eta} f_{\eta} e^{i \eta x}$, and by setting $b_{n}=\lambda-n^{2}, y_{n}=u_{\left(n+1, \eta^{\prime}\right)}$, and $f_{n}=f_{\left(n, \eta^{\prime}\right)}$, we have the recurrence formula

$$
y_{n}-b_{n} y_{n-1}+y_{n-2}=f_{n}, \quad n=0, \pm 1, \pm 2, \ldots .
$$

We first solve (4) for $n \geq-1$ with the initial condition $y_{-1}=\alpha, y_{0}=\beta$, where $\alpha$ and $\beta$ will be determined later. Then the solution is given by

$$
\begin{gathered}
y_{n}=\alpha A_{n}+\beta B_{n}+\varphi_{n}, \\
\varphi_{n}=-A_{n} \sum_{\nu=1}^{n-1} f_{\nu} B_{\nu-1}+B_{n} \sum_{\nu=1}^{n-1} f_{\nu} A_{\nu-1}+f_{n}, \\
\varphi_{-1}=\varphi_{0}=0 .
\end{gathered}
$$

So $y_{n}=\alpha A_{n}+\left(\beta+\gamma_{1}\right) B_{n}+\psi_{n}+f_{n}$, where $\gamma_{1}=\sum_{\nu=1}^{\infty} f_{\nu}\left(A_{\nu-1}-T B_{\nu-1}\right)$, $\psi_{0}=\psi_{1}=0$, and where $\psi_{n}(n \geq 3)$ are given by

$$
\psi_{n}=-\left(A_{n}-T B_{n}\right) \sum_{\nu=1}^{n-1} f_{\nu} B_{\nu-1}-B_{n} \sum_{\nu=n}^{\infty} f_{\nu}\left(A_{\nu-1}-T B_{\nu-1}\right) .
$$

We can easily show, by parts (b) and (c) of the lemma, that $\psi_{n}$ is rapidly decreasing as $n \rightarrow \infty$ because $f_{n}$ is rapidly decreasing. Since $y_{n}$ is of polynomial growth as $n \rightarrow \infty$, by assumption, it follows that

$$
\alpha T+\beta+\gamma_{1}=0 \text {. }
$$

Next we shall solve (4) for $n \leq-1$. We note that $y_{-2}=\lambda y_{-1}-y_{0}+f_{0}=$ $\lambda \alpha-\beta+f_{0}$. We replace $n$ in (4) with $-n$, and set $v_{n}=y_{-n-2}$, to obtain $v_{n}=b_{n} v_{n-1}-v_{n-2}+f_{-n}, n=1,2, \ldots ; v_{-1}=\alpha, v_{0}=\lambda \alpha-\beta+f_{0}$. We make the same arguments as above. Then we have

$$
\alpha T+\lambda \alpha+f_{0}-\beta+\gamma_{2}=0, \quad \gamma_{2}=\sum_{\nu=1}^{\infty} f_{-\nu}\left(A_{\nu-1}-T B_{\nu-1}\right) .
$$

By (7), (8), and the assumption $2 T \neq-\lambda$, we can determine $\alpha$ and $\beta$ uniquely as rapidly decreasing functions of $\eta$ as $|\eta| \rightarrow \infty$. Hence $u$ is smooth. This completes the proof.

\section{REFERENCES}

1. S. Greenfield and N. Wallach, Global hypoellipticity and Liouville numbers, Proc. Amer. Math. Soc. 31 (1972), 112-114.

2. W. B. Jones and W. J. Thron, Continued fractions, Analytic Theory and Applications, Addison-Wesley, Reading, MA, 1980. 
3. K. Taira, Le principe du maximum et l'hypoellipticité globale, Seminaire Bony-SjöstrandMeyer, no. 1, 1984/85.

4. M. Yoshino, A class of globally hypoelliptic operators on the torus, Math. Z. 201 (1989), $1-11$.

Department of Mathematics, Tokyo Metropolitan University, Fukazawa, SetagayaKU TOKYO, 158 JAPAN 